\title{
Review of: "Isotonic quadriceps endurance is better associated with daily physical activity than quadriceps strength and power in COPD: an international multicentre cross-sectional trial"
}

\author{
Isaac Selva Raj ${ }^{1}$ \\ 1 Royal Melbourne Institute of Technology
}

Potential competing interests: The author(s) declared that no potential competing interests exist.

Thank you for an excellent paper. Every query I had was answered as I read further or checked references.

The idea to study quadriceps endurance is an inspired one, and makes a lot of sense.

I think it is prudent that the authors highlighted that the current study is cross-sectional, and therefore cause and effect cannot be inferred. However, it was also useful that the authors highlight one study that showed the link between improved muscular endurance (through an exercise intervention) and increased physical activity levels.

Obviously, much more research is needed to definitively show that increased muscular endurance leads to increased physical activity levels in COPD, rather than increased muscular endurance being a 'by-product' of increased physical activity, but the current study is a good first step. 